\title{
Role of endoscopy, cross-sectional imaging and biomarkers in Crohn's disease monitoring
}

\author{
Jose-Manuel Benitez, ${ }^{1,2}$ Marie-Alice Meuwis, ${ }^{3}$ Catherine Reenaers, ${ }^{1,3}$ \\ Catherine Van Kemseke, ${ }_{1}^{1}$ Paul Meunier, ${ }^{4}$ Edouard Louis ${ }^{1,3}$
}

\begin{abstract}
${ }^{1}$ Department of
Gastroenterology, University Hospital CHU of Liège, Liège, Belgium

${ }^{2}$ Department of

Gastroenterology, University Hospital of Cordoba, Cordoba, Spain

${ }^{3}$ Gastroenterology Translational Research-GIGA Research, University of Liège, Liège Belgium

${ }^{4}$ Department of Abdominal Medical Imaging, University Hospital CHU of Liège, Liège Belgium
\end{abstract}

\section{Correspondence to}

Dr Edouard Louis, Service de Gastroentérologie, CHU de Liège, Liège 4000, Belgium; edouard.louis@ulg.ac.be

Received 2 January 2013 Revised 26 February 2013 Accepted 7 March 2013

To cite: Benitez J-M, Meuwis M-A, Reenaers C, et al. Gut 2013:62: 1806-1816.

\section{ABSTRACT}

Crohn's disease is characterised by recurrent and/or chronic inflammation of the gastrointestinal tract leading to cumulative intestinal tissue damage. Treatment tailoring to try to prevent this tissue damage as well as achieve optimal benefit/risk ratio over the whole disease course is becoming an important aspect of Crohn's disease management. For decades, clinical symptoms have been the main trigger for diagnostic procedures and treatment strategy adaptations. However, the correlation between symptoms and intestinal lesions is only weak. Furthermore, preliminary evidence suggests that a state of remission beyond the simple control of clinical symptoms, and including mucosal healing, may be associated with better disease outcome. Therefore monitoring the disease through the use of endoscopy and cross-sectional imaging is proposed. However, the degree of mucosal or bowel wall healing that needs to be reached to improve disease outcome has not been appropriately studied. Furthermore, owing to their invasive nature and cost, endoscopy and cross-sectional imaging are not optimal tools for the patients or the payers. The use of biomarkers as surrogate markers of intestinal and systemic inflammation might help. Two biomarkers have been most broadly assessed in Crohn's disease: C-reactive protein and faecal calprotectin. These markers correlate significantly with endoscopic lesions, with the risk of relapse and with response to therapy. They could be used to help make decisions about diagnostic procedures and treatment. In particular, with the use of appropriate threshold values, they could determine the need for endoscopic or medical imaging procedures to confirm the disease activity state.

\section{INTRODUCTION}

Crohn's disease (CD) is a chronic inflammatory disorder of the gastrointestinal tract leading to cumulative intestinal tissue damage and complications such as fistulas and strictures requiring surgical resection. ${ }^{1}$ A classical step-up strategy in which the treatment is adapted according to the clinical activity of the disease does not seem to be able to change the natural history of the disease, particularly to avoid the need for intestinal surgical resection. $^{2}$ Achieving mucosal healing has been associated with better patient outcome in several population-based studies and clinical trials. ${ }^{3-6}$ For these reasons and although there is no current proof that optimising therapy in a patient who has achieved clinical remission but not mucosal healing will lead to improved outcome, there is an intuitive trend to aim to achieve such healing, particularly in patients with worse prognoses. Because of the poor correlation between clinical activity of $\mathrm{CD}$ and mucosal healing, ${ }^{7}$ this requires specific monitoring, defined as the systematic use of objective tools able to assess the state of biological activity of the disease, beyond the simple assessment of clinical symptoms. Endoscopy is an invasive and costly procedure, and regular monitoring of the disease by endoscopy may not be realistic. Moreover, it is not adapted for small-bowel location above the terminal ileum, and it does not provide information about the transmural nature of the inflammation. For these locations and aspects, cross-sectional imaging may be more appropriate, but the specific features associated with tissue healing have been less well described. ${ }^{8}$ Biomarkers may represent an attractive alternative for both colonic and smallbowel disease, as they represent potential surrogate markers of intestinal inflammation and tissue lesions. Among the very large number of biomarkers assessed in $\mathrm{CD}$, the vast majority have not been adequately studied to determine their potential clinical usefulness. For most of these markers, abnormal stool or blood concentrations have been shown in CD, but no correlation has been clearly demonstrated with disease activity, intestinal lesions or disease evolution and risk of complications. The main exceptions are blood C-reactive protein (CRP) and faecal calprotectin, which have been more extensively studied, including assessment of their ability to predict response to treatment, mucosal healing, and risk of relapse and of complications. ${ }^{9}$ Depending on the clinical situation, different combinations of these imaging and biomarker monitoring tools may be used. The aim of this review is to describe the potential use, advantages and limitations of endoscopy, cross-sectional imaging and biomarkers for the monitoring of CD. We will more specifically tackle these issues through various clinical scenarios, including confirmation of disease activity, response to induction therapy, confirmation of sustained mucosal healing, and prevention of relapse or recurrence. We will not address the use of these tools for the diagnosis of $\mathrm{CD}$ or when the clinical presentation leads to the suspicion of a complication of the disease.

\section{ENDOSCOPY}

Preliminary mainly retrospective data or post hoc analyses indicate that achieving endoscopic mucosal healing may improve outcome of the disease. ${ }^{3-6}$ However, endoscopy suffers from a series of significant drawbacks: it is not that well accepted by the patients, ${ }^{10}$ although it is usually safe,${ }^{11}$ it is relatively expensive, it does not give information on the deep layers of the intestine and the extraintestinal signs of inflammation, ${ }^{12}$ and finally the 
significance of different types of lesion and the degree of endoscopic healing that should be achieved is not well established. Illustrations of unhealed, partly healed and fully healed mucosa are shown in figure 1. Advantages and drawbacks of endoscopy as a monitoring tool are summarised in table 1 . The potential role of endoscopy in monitoring patients with clinically active disease or in remission is developed in the following paragraphs and summarised in tables 4 and 5 .

\section{Confirming disease activity and severity of lesions}

The correlation between clinical activity of CD and the severity of endoscopic lesions is only weak. ${ }^{7}$ Of patients with clinically active disease, a significant proportion will have no significant endoscopic lesions. These patients do not respond optimally to the most effective treatment of $\mathrm{CD}$, such as immunosuppressant and anti-tumour necrosis factor (TNF) combination therapy. ${ }^{13}$ Preliminary data also indicate that deep colonic ulcers covering more than $10 \%$ of a colonic segment are associated with increased risk of colectomy over the next 8 years. ${ }^{14}$ Stricturing lesions have been associated with lower response rate to medical therapies and a greater need for surgery. ${ }^{15}$ To be fully informative, an endoscopic procedure for CD should thus lead to a very precise description of the type, location and extent of the lesions. Although endoscopic indexes of disease severity such as the CD Endoscopic Index of Severity (CDEIS) ${ }^{16}$ and Simplified Endoscopic Score of CD (SESCD) ${ }^{17}$ have been used in several studies, and specific thresholds of these scores have been associated with CD outcome, these thresholds have not been broadly validated. Hence, therapeutic decisions still seem to be best based on the precise description of the lesions, instead of a specific quantitative or semiquantitative threshold.

\section{Confirming mucosal healing}

Achieving endoscopic healing after medical therapy has been associated with a better disease outcome. In a population-based study from Norway, the presence of mucosal healing 1 year after the diagnosis of $\mathrm{CD}$ tended to be associated with less need for surgical resection, although this difference did not reach statistical significance. ${ }^{3}$ In the Accent 1 trial, patients achieving mucosal healing at weeks 12 and 54 experienced fewer relapses and hospitalisations. ${ }^{18}$ In the experience of a tertiary referral centre in Belgium, patients achieving at least partial healing
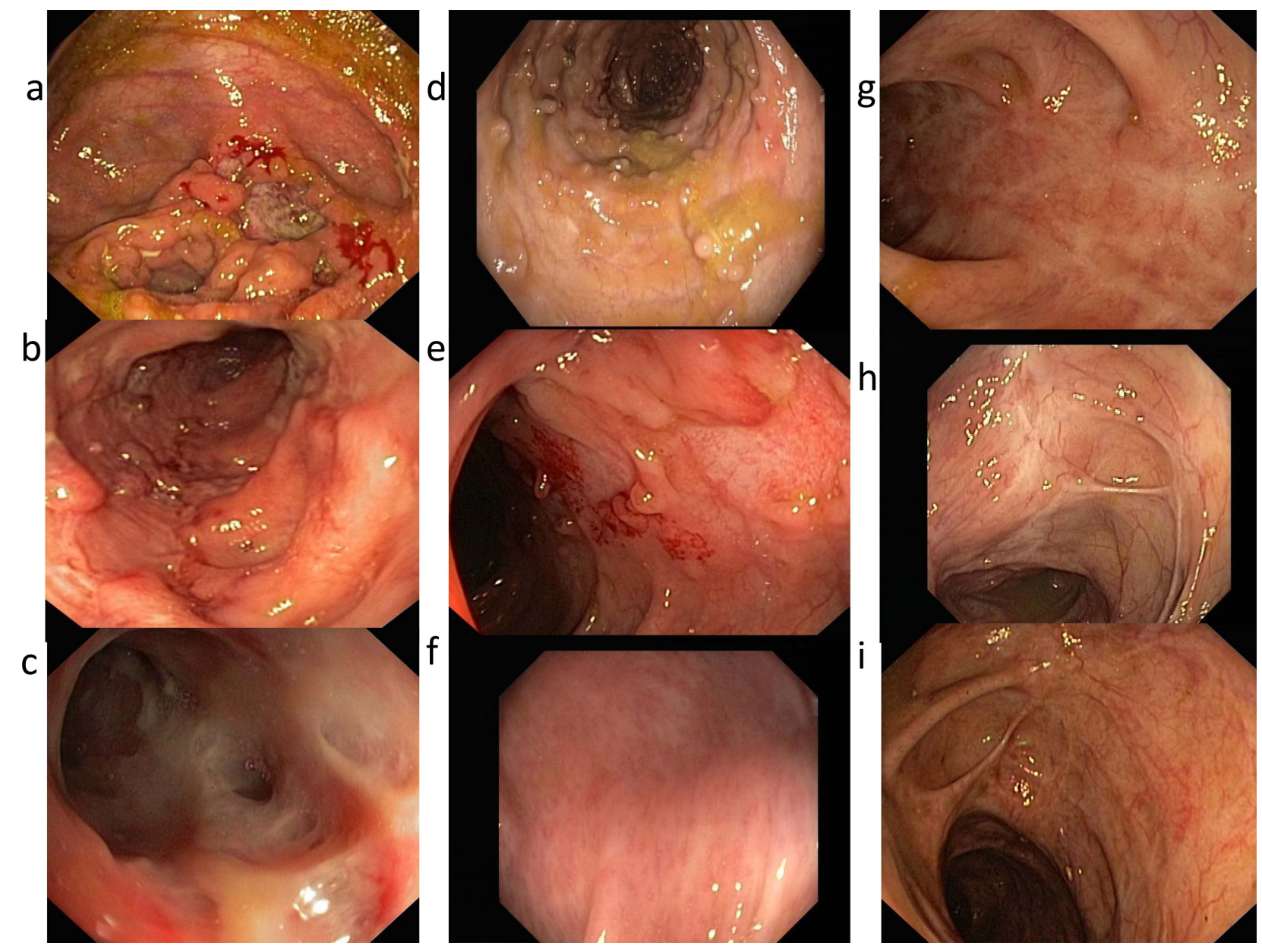

Figure 1 Examples of various degrees of endoscopic healing in Crohn's disease. (A) Absence of healing characterised by the persistence of a deep ulcer in the caecum; (B) absence of healing characterised by the persistence of extensive longitudinal and transversal ulcers in the sigmoid colon; (C) absence of healing characterised by the persistence of multiple deep ulcers in the left colon; (D) partial healing characterised by the presence of small pseudo-polyps and the persistence of focal erythema and tiny superficial ulcers in the right colon; (E) partial healing characterised by the presence of small pseudo-polyps, healed ulcers with modification of the vascular pattern, and the persistence of small superficial ulcers in the sigmoid colon; (F) partial healing characterised by the persistence of tiny aphthous lesions throughout the colon; (G) full mucosal healing characterised by the presence of longitudinal healed whitish areas; $(\mathrm{H})$ full mucosal healing characterised by the presence of whitish healed areas and mucosal bridges; (I) full mucosal healing characterised by the presence of mucosal bridges converging to a healed stellar-shaped area. 
Table 1 Monitoring of Crohn's disease with ileocolonoscopy

\begin{tabular}{ll}
\hline Advantages & Drawbacks \\
\hline Direct visualisation of & Invasive \\
intestinal lesions & Rew acceptance \\
Validated index of severity & No visualisation of the transmural \\
Predictive value for & inflammatory process \\
- Postoperative recurrence & Timing of the endoscopic monitoring \\
- Risk of relapse upon & not established \\
anti-TNF withdrawal & Degree of healing to achieve in order \\
- Risk of requiring abdominal & to affect disease outcome not \\
surgery under anti-TNF & established \\
therapy & \\
- Risk of hospitalisation under & \\
$\quad$ anti-TNF therapy & \\
\hline TNF, tumour necrosis factor. &
\end{tabular}

subsequently underwent fewer surgical resections. ${ }^{4}$ Very interestingly and intriguingly, the patients with partial healing had no more surgery than those with complete healing, emphasising the relevance of the question about the degree of healing required to improve disease outcome in CD. The long-term follow-up of the so-called 'step-up top-down trial' also revealed that patients with complete mucosal healing 2 years after the beginning of the trial (whatever the type of treatment they received) experienced fewer flares and were more often in remission without steroids and without anti-TNF over the next 2 years. ${ }^{19}$ In a controlled trial showing the superiority of adalimumab maintenance over placebo to achieve early and sustained mucosal healing in $\mathrm{CD}$, the patients with mucosal healing after 12 weeks had fewer relapses and hospitalisations over the 1-year follow-up. ${ }^{6}$ On the basis of these preliminary data, endoscopic monitoring after induction or during maintenance therapy is now advocated by many experts. However, there are currently no data to clarify the timing of this monitoring, the degree of healing that needs to be reached, and, above all, the management of insufficiently healed patients. No prospective or even retrospective study can report on an improved outcome in these unhealed patients after a change in therapy. This is even more troubling because the only available data in this field, dating back to the time of corticosteroid therapy, do not support such treatment optimisation. Indeed, in the 1990s, a GETAID Study showed that prolonging steroid treatment in patients in clinical remission but with unhealed mucosa after 6 weeks of full steroid induction did not improve the relapse rate over 1 year, despite a slight increase in the proportion of patients whose mucosa was healed. ${ }^{20}$ Because of all these unsettled issues, the use of endoscopic monitoring to confirm tissue healing in $\mathrm{CD}$ can only be empirical. An endoscopy should only be discussed if a clear disease management plan can be proposed to the patient depending on the results of this endoscopy. In our view, this could be particularly adapted when there is concern about disease progression and its consequences. In some situations, such as extensive small-bowel disease, previous multiple intestinal resections, deep and extensive colonic (particularly sigmoid or rectal) ulcers, due to the consequences of uncontrolled disease, endoscopic monitoring and treatment optimisation in the case of persisting significant lesions may be proposed.

\section{Assessing the patient before therapy de-escalation}

In order to achieve optimal benefit/cost and benefit/risk ratio for the patient, treatment de-escalation in patients who have reached sustained remission may be as important as treatment optimisation in those who have not reached this target. Whether treatment de-escalation is possible in a subset of patients and what the criteria should be is not precisely known. However, preliminary results are provided by a prospective cohort study from the GETAID. In this study, full endoscopic healing was associated with a lower risk of relapse after infliximab withdrawal in patients treated with immunosuppressant/ infliximab combination therapy for more than 1 year and in steroid-free remission for more than 6 months. ${ }^{21}$ In patients without mucosal healing, defined by a CDEIS $>0$, the relapse rate over 1 year was above $60 \%$. Therefore endoscopic exploration could be advocated in these patients before a decision is made on such drug withdrawal. However, mucosal healing is not the best individual predictor, and the prediction is significantly improved if some demographic characteristics, blood tests and faecal calprotectin are integrated.

\section{Predicting postoperative recurrence}

The postoperative setting is a situation where the use of endoscopic monitoring has been particularly widely used in routine practice. The disease is usually clinically quiescent, but the recurrence rate is known to be very high. This recurrence has been well described in the seminal paper by Rutgeerts et al. ${ }^{22}$ Over 8 years after an ileocolonic resection, $~ 90 \%$ experienced endoscopic recurrence, $60 \%$ clinical recurrence and 30\% surgical recurrence. This study also showed that the clinical recurrence rate was strongly associated with endoscopic recurrence within 1 year of ileocolonic resection. Diffuse ileitis, strictures and large or deep ulcers (Rutgeerts scores i3 and i4) were associated with almost $90 \%$ of clinical relapse over 8 years, while no lesion or a few aphthoid ulcers (Rutgeerts scores i0 and i1) were associated with a clinical relapse rate of $\sim 10 \%$. Following these results, it has become common practice to perform

Table 2 Monitoring of CD with cross-sectional imaging

Advantages
- Visualisation of the small bowel
Possibility of visualisation of the small bowel and the colon in one procedure with MRI
enterocolonography


Table 3 Monitoring of Crohn's disease with blood C-reactive protein and faecal calprotectin

\begin{tabular}{|c|c|}
\hline Advantages & Drawbacks \\
\hline $\begin{array}{l}\text { Non-invasive } \\
\text { Good acceptance } \\
\text { Relatively low cost } \\
\text { Can be repeated as a } \\
\text { longitudinal monitoring tool } \\
\text { May be combined to } \\
\text { improve prediction } \\
\text { Predictive value for } \\
\text { - Disease relapse under or } \\
\text { after medical therapy } \\
\text { - Response to anti-TNF } \\
\text { treatment } \\
\text { - Mucosal healing }\end{array}$ & $\begin{array}{l}\text { Subject to non-specific variations } \\
\text { Stool marker not always well accepted } \\
\text { by patients (faecal calprotectin) } \\
\text { The correlation with mucosal healing } \\
\text { and transmural healing is imperfect } \\
\text { Predictive threshold values not fully } \\
\text { established }\end{array}$ \\
\hline
\end{tabular}

ileocolonoscopic surveillance once, between 6-12 months after ileocolonic resection in order to adapt treatment according to the results.

\section{Potential role for small-bowel capsule endoscopy}

Small-bowel capsule endoscopy may have much better acceptance than classical endoscopic explorations. It can also visualise the whole small bowel, and its diagnostic yield in small-bowel lesions of CD has been well established. ${ }^{23}$ It could thus be of help in assessing disease activity or mucosal healing in the small bowel. However, it is hampered by a relatively high retention rate in $\mathrm{CD}$, above $10 \% .^{23}$ Although this can usually be avoided by a test with a patency capsule, obstructive accidents may still occur. Furthermore, for this disease location, it is in competition with cross-sectional imaging techniques (see below), which may also offer information on transmural inflammation and assess stricturing and fistulising complications. ${ }^{8}$ Some lesions missed at cross-sectional imaging may be diagnosed by capsule endoscopy, particularly in the proximal small bowel, but their clinical significance is still unclear. ${ }^{24}$ Several small-bowel capsule endoscopy indexes of severity have been proposed but not yet broadly validated. ${ }^{23}$

Small-bowel capsule endoscopy has been specifically assessed in the postoperative recurrence setting. Its correlation with ileocolonoscopy appears to be good, and it may thus represent an alternative in this situation. ${ }^{23}$

\section{CROSS-SECTIONAL IMAGING TECHNIQUES FOR THE MONITORING OF CD}

Although it may currently be considered the gold standard, the monitoring of $\mathrm{CD}$ activity and mucosal healing by ileocolonoscopy or small-bowel capsule endoscopy is hampered by a series of drawbacks highlighted above. Therefore the use of crosssectional imaging techniques to monitor the disease is rapidly increasing. ${ }^{8}$ These techniques, including ultrasonography (US), CT and MRI, show both parietal and extraparietal changes caused by the disease, and allow evaluation of small-intestinal regions inaccessible to ileocolonoscopy, enabling the identification of a whole spectrum of lesions with good resolution. ${ }^{8}$

MRI is considered the standard imaging technique for assessment in patients with $\mathrm{CD}$ who require many follow-up examinations and are usually a young population. ${ }^{25}$ The absence of ionising radiation, along with very high soft-tissue contrast, multiplanar images, low incidence of adverse events related to the intravenous contrast, and high diagnostic accuracy in the evaluation of luminal and extraluminal abnormalities, justify its application. ${ }^{26} \mathrm{CT}$ has a similar accuracy to MRI for assessing bowel damage in $\mathrm{CD},{ }^{27}$ but the risk of radiation exposure should limit its use as a monitoring tool. US is another non-ionising alternative, ${ }^{28}$ but has some drawbacks such as the difficulty of visualising deep bowel segments, high interobserver variability, and often incomplete exploration due to gas interposition. ${ }^{29}$

An illustration of monitoring of multifocal small-bowel CD with MR enterography is shown in figure 2. Advantages and drawbacks of cross-sectional imaging as a monitoring tool are summarised in table 2 . The potential role of cross-sectional imaging in monitoring patients with clinically active disease or in remission is developed in the following paragraphs and summarised in tables 4 and 5 .

\section{Assessment of disease activity and severity and response to treatment}

The high accuracy of MR enteroclysis, enterography and enterocolonography has been demonstrated in the assessment of activity and severity in $\mathrm{CD}$, with several parameters related to the degree of activity being identified. ${ }^{30}$ Globally, enterography has been preferred to enteroclysis because of its better acceptance. Enterocolonography implies a colonic distension by enema and may provide information on the whole gastrointestinal tract in only one procedure. The main MRI findings that correlate with intestinal inflammation and disease activity and severity include wall thickening, bowel wall enhancement, mural oedema (signal hyperintensity on T2-weighted sequences) and presence of ulcers. It is in the terminal ileum and the colon that the ability

Table 4 Potential monitoring of a patient with clinically active CD

\begin{tabular}{|c|c|c|}
\hline Question & Monitoring tool & Prediction \\
\hline Are there endoscopic active lesions? & $\begin{array}{l}\text { CRP } \leq 5 \mathrm{mg} / \mathrm{l} \text { and faecal calprotectin } \\
\leq 200 \mu \mathrm{g} / \mathrm{g} \\
\text { MR enterocolonography (MaRIA } \\
\text { score) }\end{array}$ & $\begin{array}{l}\text { Predicts CDEIS } \leq 6 \text { with a sensitivity of } 83 \% \text { and specificity of } 71 \%{ }^{57} \\
\text { Predicts active endoscopic ileocolonic lesions with a sensitivity of } 87 \% \text { and a } \\
\text { specificity of } 89 \% 31\end{array}$ \\
\hline $\begin{array}{l}\text { Is the patient going to respond to anti-TNF } \\
\text { therapy (before induction)? }\end{array}$ & $\begin{array}{l}\text { CRP }>5 \mathrm{mg} / \mathrm{l} \\
\text { Presence of any ulcer at } \\
\text { ileocolonoscopy }\end{array}$ & $\begin{array}{l}76 \% \text { response rate after first anti-TNF (compared with } 46 \% \text { when normal CRP) } \\
61 \% \text { steroid-free remission with azathioprine+infliximab combination therapy } \\
\text { (compared with } 40 \% \text { without endoscopic lesion) }\end{array}$ \\
\hline $\begin{array}{l}\text { Is the patient going to respond to anti-TNF } \\
\text { therapy (after induction)? }\end{array}$ & $\begin{array}{l}\text { Faecal calprotectin decrease } \\
\text { Absence of sustained CRP } \\
\text { normalisation }\end{array}$ & $\begin{array}{l}\text { Increased proportion of endoscopic healing } \\
\text { Increased risk of anti-TNF loss of response }\end{array}$ \\
\hline
\end{tabular}


Table 5 Potential monitoring of patients with $C D$ in clinical remission

\begin{tabular}{|c|c|c|}
\hline Question & Monitoring tool & Prediction $(95 \% \mathrm{Cl})$ \\
\hline $\begin{array}{l}\text { Has mucosal healing been } \\
\text { achieved? }\end{array}$ & $\begin{array}{l}\text { CRP } \leq 10 \mathrm{mg} / \mathrm{l} \text { and faecal calprotectin } \\
\leq 200 \mu \mathrm{g} / \mathrm{g}\end{array}$ & CDEIS $\leq 3$ with a sensitivity of $78 \%$ and specificity of $58 \%{ }^{57}$ \\
\hline What is the risk of relapse? & $\begin{array}{l}\text { Increased CRP } \\
\text { Increased faecal calprotectin } \\
\text { Mucosal healing at ileocolonoscopy } \\
(\mathrm{SESCD}=0)\end{array}$ & $\begin{array}{l}\text { Relative risk of relapse increasing to } 3-58^{* 70-72} \\
\text { Clinical relapse over } 1 \text { year with a sensitivity of } 43-90 \% \text { and a specificity of } \\
43-88 \% \text { (depending on the threshold for faecal calprotectin**67-69 } \\
69 \% \text { of remission without steroids over the next } 2 \text { years (compared with } 38 \% \text { if no } \\
\text { mucosal healing) })^{19}\end{array}$ \\
\hline $\begin{array}{l}\text { What is the risk of abdominal } \\
\text { surgery? }\end{array}$ & $\begin{array}{l}\text { Mucosal healing at ileocolonoscopy (at least } \\
\text { partial) }\end{array}$ & $14 \%$ requiring abdominal surgery (vs $38 \%$ if absence of healing) ${ }^{4}$ \\
\hline $\begin{array}{l}\text { What is the risk of relapse upon } \\
\text { anti-TNF withdrawal? }\end{array}$ & $\begin{array}{l}\text { CRP } \geq 5 \mathrm{mg} / \mathrm{l} \\
\text { Faecal calprotectin } \geq 300 \mu \mathrm{g} / \mathrm{g} \\
\text { Absence of mucosal healing at } \\
\text { ileocolonoscopy }(\text { CDEIS }>0)\end{array}$ & $\begin{array}{l}\text { HR for relapse } 2.5(1.4-4.4)^{21} \\
\text { HR for relapse } 3.2(1.7-6.2)^{21} \\
\text { HR for relapse } 1.8(1.0-3.3)^{21}\end{array}$ \\
\hline $\begin{array}{l}\text { What is the risk of postoperative } \\
\text { clinical recurrence? }\end{array}$ & $\begin{array}{l}\text { Endoscopic Rutgeerts score within } \\
6-12 \text { months after surgical resection }\end{array}$ & $\begin{array}{l}\text { i0-i1: } 10 \% \text { of relapse at } 8 \text { years }^{22} \\
\text { i2: } 40 \% \text { of relapse at } 8 \text { yearss } \\
\text { i3-i4: } 90 \% \text { of relapse at } 8 \text { years }\end{array}$ \\
\hline
\end{tabular}

${ }^{*}$ Increase in CRP and faecal calprotectin occurs within the 4-6 months before relapse. ${ }^{73}$

CD, Crohn's disease; CDEIS, CD Endoscopic Index of Severity; CRP, C-reactive protein; SESCD, Simplified Endoscopic Score of CD; TNF, tumour necrosis factor.

a

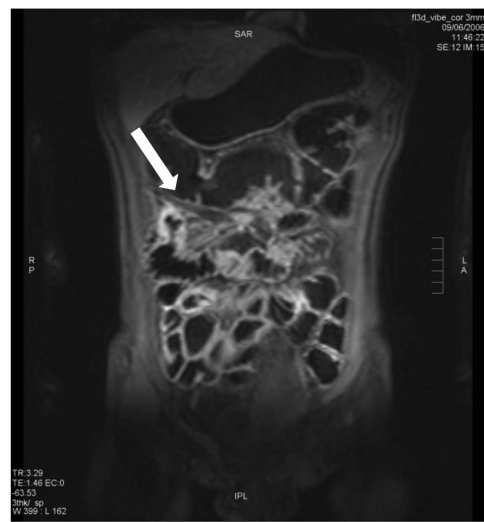

b

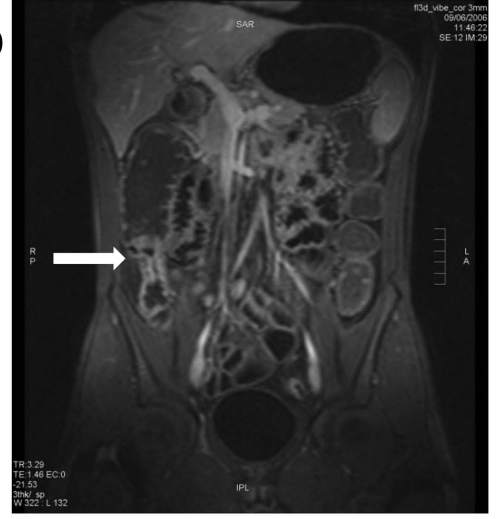

$9 / 6 / 2006$
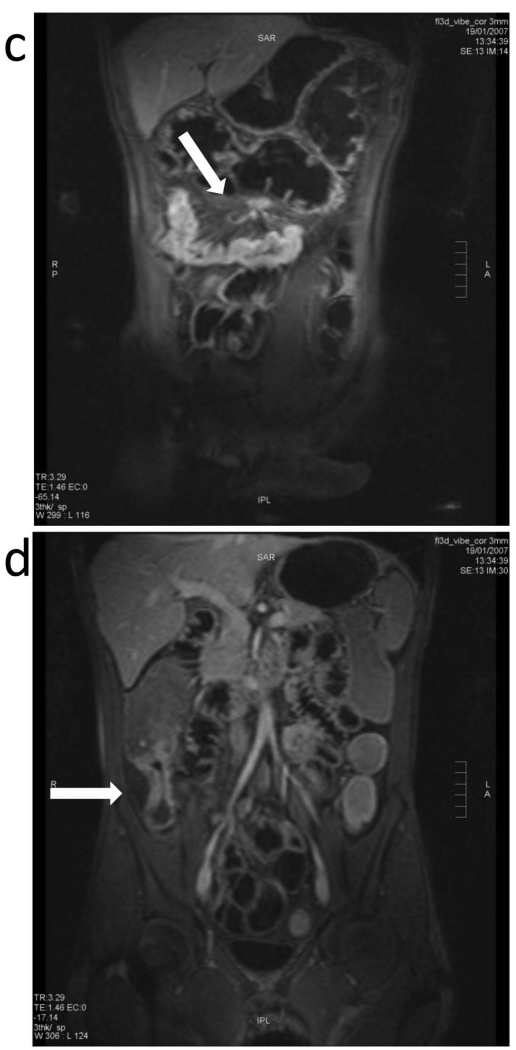

19/1/2007

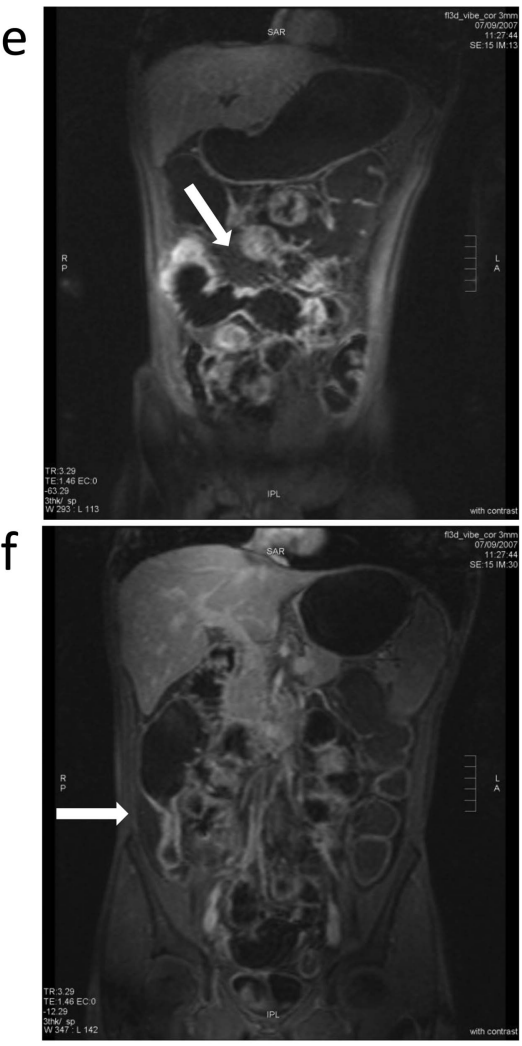

$7 / 9 / 2007$

Figure 2 Example of monitoring of small-bowel Crohn's disease (CD) with MR enterography. This patient had been operated on three times for recurring stricturing and occlusive CD in December 1998, March 2002 and December 2005. He had been continuously treated with azathioprine between the second and third operations. He was prescribed methotrexate $15 \mathrm{mg} /$ week subcutaneously immediately after the third operation. Six months later, in June 2006, he was in clinical remission and had a normal C-reactive protein (CRP) concentration. Because of the multifocal small-bowel CD, it was decided to monitor him with MR enterography instead of colonoscopy. The MR enterography performed in June 2006 showed thickening of the bowel wall without strong contrast enhancement at the ileocolonic anastomosis $(B)$ and mild thickening and wall enhancement in a jejunal segment (A). In January 2007, the patient was still in clinical remission with a normal CRP concentration, but had mild iron-deficiency anaemia (haemoglobin concentration $11.7 \mathrm{mg} / \mathrm{dl}$ ). The MR enterography performed at that stage showed a stable lesion at the ileocolonic anastomosis (D) and marked contrast enhancement and thickening of the wall in the jejunal segment, together with hyperaemia of the vasa recta (comb sign) (C). The patient was then treated with infliximab in combination with methotrexate. In September 2007, the patient was still in clinical remission with normal CRP concentration. The anaemia had disappeared. The MR enterography showed a significant decrease in wall thickening and contrast enhancement of the jejunal segment, but a partial small-bowel obstruction due to jejunal disease (E), while the anastomotic stricture remained unchanged (F). Maintenance treatment combining methotrexate $15 \mathrm{mg} /$ week orally and infliximab $5 \mathrm{mg} / \mathrm{kg}$ every 8 weeks was prescribed . 


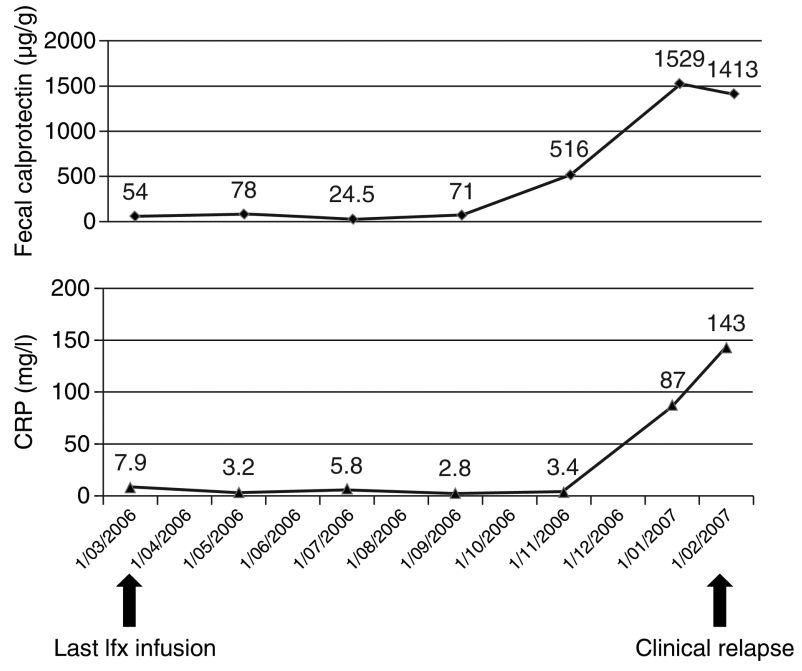

Figure 3 Example of monitoring a patient with Crohn's disease (CD) in clinical remission by using blood C-reactive protein (CRP) and faecal calprotectin. This patient had been treated with azathioprine/infliximab combination therapy since July 2003 for ileocolonic refractory CD. In March 2006, he had been in stable steroid-free remission for more than 1 year. Following a request from the patient, it was decided to withdraw infliximab and to continue with azathioprine monotherapy. The last infliximab (Ifx) infusion was administered on 15 March 2006. At that time, CRP was slightly increased at $7.9 \mathrm{mg} / \mathrm{l}$, but faecal calprotectin was low at $54 \mu \mathrm{g} / \mathrm{g}$. CRP and faecal calprotectin were measured every 2 months after the last infliximab infusion. While the patient was still in remission in November 2006, a significant increase in faecal calprotectin was noticed $(516 \mu \mathrm{g} / \mathrm{g})$, whereas CRP was still normal (3.4 mg/l). Two months later, faecal calprotectin had further increased to $1529 \mu \mathrm{g} / \mathrm{g}$, and CRP had also significantly increased to $87 \mathrm{mg} / \mathrm{l}$. Clinical relapse finally occurred 1 month later in February 2007.

of MRI to assess disease activity has been best validated. Using the above parameters, Rimola et $a l^{30} 31$ proposed and validated a simplified Magnetic Resonance Index of Activity (MaRIA) score to quantify disease activity based on MRI findings in each ileocolonic segment, which strongly correlated with CDEIS. For the detection of active CD in the terminal ileum and the colon, the sensitivity of this score was $87 \%$, specificity $89 \%$, positive predictive value (PPV) 98\%, negative predictive value (NPV) $88 \%$ and overall accuracy $98 \%$. This score was also able to accurately predict severely ulcerated CD. Therefore, the MaRIA score represents an objective, quantitative and reproducible measure of activity and could categorise disease severity and monitor response to therapeutic interventions in the terminal ileum and the colon.

These results agree with those from other studies identifying MRI signs associated with pathological inflammation mainly in the small bowel, using surgical examination as a reference method. ${ }^{32} 33$ In a systematic review, Panes et $a l^{8}$ reported a sensitivity and specificity of MRI for the assessment of disease activity on a per patient basis of $80 \%$ and $82 \%$, respectively. The use of oral and intravenous contrast agent promotes bowel lumen distension and improves detection of these features. ${ }^{34}$ CT has a similar accuracy to MRI for distinguishing activity in the terminal ileum, with a sensitivity of $81 \%$ and specificity of $88 \%{ }^{83536}$

US has been established as a reliable imaging technique for the assessment of disease activity in CD. The wall thickness and vascularisation pattern shown by Doppler US are particularly useful for the detection of active disease. The overall sensitivity and specificity of US for detecting disease activity have been found to be $\sim 85 \%$ and $\sim 91 \%$, respectively, ${ }^{8}$ although its performance is largely dependent on the operator and the disease location. $^{37}$ The use of contrast-enhanced US seems to increase its accuracy for the evaluation of activity in CD, with a sensitivity of $93 \%$ and specificity of $94 \% .^{38}$ It also seems to better classify disease severity than Doppler US signal and measurement of wall thickening.

Few studies have investigated the capability of MRI for assessing treatment response after a flare of CD. Sempere et al ${ }^{39}$ evaluated this capability of MRI compared with ileocolonoscopy in patients in the active phase of disease or in remission and in healthy controls. These authors reported that contrast enhancement of the bowel wall decreased significantly from the active to the remission phase. The mean contrast enhancement in active $C D$ was significantly greater than in the control group, although there was no difference between patients in remission and the healthy controls. The same occurred with bowel wall thickness, which was significantly decreased in the remission phase. However, the segments remained thickened in patients with CD compared with healthy controls. This is probably due to a double component: an acute factor (inflammation and oedema) and a chronic factor (fibrosis), which may not reverse despite therapeutic response. Another study has evaluated MRI for monitoring therapeutic responses using the MaRIA score and ileocolonoscopy as a reference standard. In this setting, the MaRIA score predicted endoscopic remission with a sensitivity of $82 \%$ and specificity of $85 \% .^{40}$

All these data suggest that cross-sectional imaging could be used for the assessment of disease activity and response to therapy in CD. The use of a CT scanner should be avoided in this setting to minimise irradiation. MRI enterography could be used essentially to visualise small-bowel CD not evaluable by standard endoscopic procedures. MRI enterocolonography, could be used as a single exploration of the whole gastrointestinal tract, particularly in ileocolonic disease, decreasing the need for supplementary colonoscopies. This would be of particular interest for patients with both small-bowel and colonic lesions. US is an attractive alternative, particularly in attempts to confirm active disease. However, it is less powerful than MRI for broadly assessing lesion extent and severity, because of its poorer performance in the colon and inability to systematically see all intestinal segments.

\section{Detection of complications}

Chronic intestinal inflammation in CD can result in complications during the course of the disease. Although they may present as acute modifications of the clinical situation leading to emergency work up, they can also develop more silently, and their identification through disease monitoring may reveal disease aggressiveness and influence treatment strategy. The ability of cross-sectional imaging methods to demonstrate extramural changes makes them accurate procedures for detecting complications.

US, CT and MRI have a high sensitivity and specificity for the diagnosis of stenosis affecting the large or small bowel ${ }^{8}$ identified as an intestinal loop with wall thickening, narrowed lumen and prestenotic dilation. A systematic review of pooled results from seven studies reported that MRI had a sensitivity for detection of stenosis of $\sim 89 \%$ and a specificity of $\sim 94 \% .^{8}$ This is usually considered to be slightly higher than with CT or US. Distinguishing between inflammatory and fibrotic strictures is important, as it may have a significant effect on response to medical treatments. Making an exclusive distinction between an 
inflammatory or fibrotic pattern is difficult as they usually coexist, especially in patients with severe disease. ${ }^{41}$ Nevertheless, here again, MRI may be of help. Collagen deposition in the bowel wall is known to result in late gadolinium enhancement. A decrease in the signal intensity of the thickened wall and a reduction in bowel-wall early contrast enhancement are usually related to intestinal fibrosis. ${ }^{42}$

The diagnostic accuracy of MRI for intra-abdominal fistulas has been evaluated in multiple studies. ${ }^{8}$ Pooled results showed a sensitivity of $\sim 76 \%$ and a specificity of $\sim 96 \%$. CT has a similar accuracy, ${ }^{43}$ while US is significantly less efficient. ${ }^{8}$ Likewise, MRI detected intra-abdominal abscesses with a sensitivity ranging from $86 \%$ to $100 \%$, and specificity from $93 \%$ to $100 \%{ }^{27}$ With CT, the sensitivity was $84 \%$ and specificity $97 \% .{ }^{8}$ The value of US for detection of abscesses reached a sensitivity of $81-100 \%$ and specificity of $92-94 \% .{ }^{8}$ However, US accuracy was highly related to disease location, ${ }^{44}$ and its diagnostic accuracy was slightly lower than that of CT and MRI because of false-positive cases. Combining CT with US did not significantly improve their diagnostic accuracy for detection of abscesses in CD. ${ }^{45}$

\section{Assessment of postoperative disease recurrence}

Although ileocolonoscopy is the gold standard technique for evaluating recurrence of $\mathrm{CD}$ after intestinal resection, MRI and US may be valuable alternatives to avoid repeated colonoscopies. An MRI-based score has even been validated for the detection of postoperative recurrence compared with endoscopic Rutgeerts score. ${ }^{4647}$ Mild bowel-wall thickening and enhancement without stricture were considered to be signs of low-grade recurrence. In contrast, the presence of a clear stricture and increased bowel-wall thickness and enhancement correlated with severe recurrence. The sensitivity and specificity of MRI for detecting moderate to severe recurrence were $100 \%$ and $89 \%$, respectively.

\section{Monitoring tissue damage}

Tissue damage in CD is characterised by intestinal resections, strictures and fistulising lesions. ${ }^{48}$ One of the main aims of optimised therapeutic strategies for $\mathrm{CD}$, including early intensive treatment and tight disease control, is to limit, or even suppress, the development of tissue damage. Owing to its ability to visualise and quantify both stricturing and penetrating lesions of CD, MRI currently represents the best candidate for monitoring tissue damage in CD. A tissue damage score is currently under development. $^{48}$

\section{BIOMARKERS}

CRP is an acute phase reactant produced by the liver. ${ }^{49}$ In CD, there is also significant CRP production by the mesenterium itself. $^{50}$ The main trigger for CRP production is interleukin $6 .^{51}$ In $\mathrm{CD}$, interleukin 6 is produced in the whole intestinal wall and probably the mesenterium, at the site of inflammation, by a broad selection of cell types including lymphocytes, monocytes, granulocytes, fibroblasts, and epithelial and endothelial cells. CRP increases very rapidly during acute inflammation and may remain elevated during chronic inflammation.

Calprotectin is a heterodimer or heterotetramer combining S100A8 and S100A9 proteins. $^{52}$ It is produced at the site of inflammation mainly by granulocytes, but also monocytes and epithelial cells. Owing to this direct production by intestinal epithelial cells, increased amounts of calprotectin may be found in the stool even in cases of mild mucosal inflammation. ${ }^{53}$ Production and secretion of calprotectin is activated by stimulating producing cells by inflammatory cytokines such as interleukin 1, activated complement, immunoglobulins through Fc receptor binding, and bacterial products such as lipopolysaccharide.

Monitoring of a patient with CD in remission with blood CRP and faecal calprotectin is illustrated in figure 3. Advantages and drawbacks of blood CRP and faecal calprotectin as monitoring tools are summarised in table 3 . Their potential roles in monitoring patients with clinically active $\mathrm{CD}$ or in remission is developed in the following paragraphs and summarised in tables 4 and 5. Other blood and faecal biomarkers, such as faecal lactoferrin, show promise as potential biomarkers for CD, but have been studied far less extensively. ${ }^{9}$ Their place in CD monitoring cannot yet be discussed.

\section{Biomarkers to confirm disease activity and assess response to treatment}

The importance of confirming inflammatory activity in a patient with clinical symptoms before starting or escalating medical treatment for $\mathrm{CD}$ has been highlighted above in the discussion of monitoring by endoscopy and cross-sectional imaging. Blood markers could be of help here to avoid repeating these invasive procedures. Another feature is rapid confirmation of drug efficacy when the treatment has been started. Here again the early repetition of endoscopy and/or cross-sectional imaging is inappropriate, and blood markers could provide important information.

\section{Confirming disease activity before treatment}

Although it does not correlate perfectly with endoscopic scores of activity, CRP represents an objective marker of active inflammation. Response to medical therapy for CD, particularly anti-TNF antibodies, has been shown to be better when an increase in CRP was present to confirm disease activity. ${ }^{54}$ In contrast, in patients with a normal CRP despite clinical activity of the disease, a substantial proportion of patients may have functional disorders or the aftermath of previous flares or surgeries. A prospective study has specifically addressed this point in patients with a CDAI $>150$ but a CRP $<5 \mathrm{mg} / \mathrm{l}$. A colonoscopy was systematically performed; this showed only minor lesions in the majority of the patients, but still one-third of them had a CDEIS $>6$, confirming clinically significant lesions. ${ }^{55}$ From this, it seems logical to advocate controlling endoscopy or medical imaging for signs of disease activity in patients with clinically active disease but a normal CRP concentration. Faecal calprotectin correlates better with endoscopic scores of disease severity, with a correlation coefficient of $\sim 0.70 .^{56}$ Although it may represent a more efficient marker than CRP in this setting, this correlation is still imperfect. CRP and faecal calprotectin may thus be best used as first-line tools to decide whether or not there is a need for endoscopic or cross-sectional imaging reassessment. In this perspective, an interesting meta-study was recently performed, analysing the data of six studies that included more than 550 patients and provided blood CRP and faecal calprotectin levels together with endoscopic scores of severity. ${ }^{57}$ In patients with symptoms (CDAI >220), the sensitivity of CRP $\leq 5 \mathrm{mg} / \mathrm{l}$ or calprotectin $\leq 200 \mu \mathrm{g} / \mathrm{g}$ to anticipate a CDEIS $\leq 6$ was $83 \%$ and the specificity $71 \%$. The PPV ranged from $66 \%$ to $81 \%$ and NPV from $86 \%$ to $73 \%$ for a prevalence of CDEIS $\leq 6$ between $40 \%$ and $60 \%$. It thus means that, out of 100 patients with clinically active disease, if endoscopy was only performed when one of the markers was below the threshold, 38-50 colonoscopies could be avoided. Those with both markers above the threshold would be considered to have active lesions. However, 7-10 of them would have a CDEIS $<6$ and may thus be overtreated. 
Confirming response to treatment

A decrease in CRP has been clearly demonstrated in patients clinically responding to medical treatment. The same has been shown for faecal calprotectin. ${ }^{58}$ More recently, it was shown that a persisting increase in CRP under anti-TNF therapy was associated with future loss of response to the drug. ${ }^{59}$ This suggests that biomarkers such as CRP and faecal calprotectin could be used to confirm the response to therapy and that an adequate response should be accompanied by normalisation of CRP and a dramatic decrease in faecal calprotectin. Normalisation of calprotectin $(<50 \mu \mathrm{g} / \mathrm{g})$ is certainly more difficult to achieve than normalisation of CRP, and may not be considered as a therapeutic target. Nevertheless, it may represent a state of deeper remission, probably associated with more profound mucosal healing. ${ }^{21}$

\section{Biomarkers to confirm tissue healing and predict disease relapse}

Correlation coefficients between faecal calprotectin and endoscopic scores of disease activity ranged from 0.42 to 0.73 , and the sensitivity and specificity to predict absence of mucosal healing were $70-100 \%$ and $44-100 \%$, respectively, depending on the calprotectin concentration threshold used. ${ }^{56}{ }^{60-62}$ In the largest study so far, a faecal calprotectin concentration $>250 \mu \mathrm{g} /$ g predicted large ulcers with a sensitivity of $60 \%$ and a specificity of $80 \%$, and a concentration $<250 \mu \mathrm{g} / \mathrm{g}$ predicted mucosal healing (CDEIS <3) with a sensitivity of $94 \%$ and specificity of $62 \% .{ }^{63}$ The weaknesses of faecal calprotectin as a biomarker may be a weaker correlation with endoscopic activity in the small bowel, ${ }^{60}$ the imperfect reflection of the transmural inflammatory process, and finally the unpleasant requirement for the patient to bring a stool sample to the laboratory. While no other marker has been specifically studied for the assessment of smallbowel mucosal inflammation, the transmural process may be better reflected by CRP. Indeed, in a retrospective study assessing correlation between CRP concentration and various semiological features at CT cross-sectional imaging, CRP correlated more closely with transmural and mesenteric signs of inflammation than with purely mucosal signs of inflammation. ${ }^{64}$ In some studies, the correlation between serum inflammatory markers, including CRP, and endoscopic activity of the disease was close to that of faecal calprotectin, with a correlation coefficient of $\sim 0.70 .^{65}$ For the assessment of mucosal healing, a recent subanalysis of the STORI cohort suggested that the combination of faecal calprotectin (at a threshold of $250 \mu \mathrm{g} / \mathrm{g}$ ) and CRP (at a threshold of $5 \mathrm{mg} / \mathrm{l}$ ) may improve the ability to predict such healing by significantly increasing specificity above $70 \%$ while sensitivity remained reasonably good, also above $70 \% .{ }^{66}$ As for the prediction of endoscopically active disease, the prediction of mucosal healing with biomarkers is imperfect, with an inaccuracy of $30-40 \%$. It is thus again as first-line tests and in combination with endoscopy and/or cross-sectional imaging that these biomarkers may be best used. The previously mentioned meta-study also addressed this question. ${ }^{57}$ When patients with inactive disease (CDAI $\leq 150)$ are considered, the sensitivity of the association of CRP $\leq 10 \mathrm{mg} / \mathrm{l}$ and calprotectin $\leq 200 \mu \mathrm{g} / \mathrm{g}$ to predict a CDEIS $\leq 3$ was $78 \%$ and the specificity $58 \%$. The PPV ranged from $65 \%$ to $88 \%$ and NPV from $73 \%$ to $40 \%$ for a prevalence of CDEIS $\leq 3$ between $50 \%$ and $80 \%$. This means that, out of 100 patients in clinical remission, if endoscopy was only performed when both markers were below the thresholds, 30-40 colonoscopies could be avoided. Patients with CRP or calprotectin greater than the threshold would be considered to have active lesions. Of these 60-70 patients, only 11-18 would have a CDEIS $\leq 3$ and would thus risk overtreatment.

CRP and faecal calprotectin are also predictors of CD relapse. Globally, the sensitivity and specificity of faecal calprotectin to predict CD clinical relapse was $43-90 \%$ and $43-88 \%$, respectively, again depending on the threshold used. ${ }^{67-69}$ In various studies, CRP was, overall, a less powerful predictor, although increased CRP was associated with a significant increase in relapse risk, the relative risk ranging between 3 and $58 .^{70-72}$ The frequency with which these biomarkers should be measured in the follow-up of patients who have achieved clinical remission is not well established. Most studies measured these markers only once and then assessed the time to relapse or the relapse rate over 1 year. However, preliminary data from the GETAID STORI cohort indicate that both CRP and faecal calprotectin start to increase 4-6 months before the relapse, and thus measurement of these markers every 3-4 months should be able to catch this increase and enable the clinician to adapt the therapeutic strategy. ${ }^{73}$

As emphasised above, in patients with longstanding stable steroid-free remission, treatment de-escalation may be contemplated to try to optimise benefit/risk and benefit/cost ratio. The data from the STORI cohort indicate that biomarkers, particularly CRP and faecal calprotectin, may be complementary to endoscopic assessment in predicting the risk of relapse upon infliximab withdrawal. $^{21}$ Increased CRP has already been associated with increased risk of relapse after azathioprine withdrawal. ${ }^{74}$

Biomarkers have been little studied in the postoperative setting to try to predict disease recurrence. Preliminary data indicate that CRP is probably not sensitive enough to be useful in this clinical setting. ${ }^{75}$ A postoperative follow-up study clearly showed the absence of correlation between endoscopic recurrence and CRP. Inflammation at this stage may be confined to the mucosa in many cases and badly translate into systemic inflammation. Faecal calprotectin seems to be much more promising. A surgical series showed normalisation of faecal calprotectin in all patients after uncomplicated curative surgery within 2 month of the resection. ${ }^{76}$ This marker remained low in patients without relapse, while it increased significantly in patients experiencing clinical relapse. However, the precise timing of this increase was not specifically assessed in this study and thus the predictive ability of faecal calprotectin in that setting cannot be determined.

\section{CONCLUSION}

Endoscopic techniques, cross-sectional imaging and biomarkers represent a range of potential monitoring tools for CD. They all have theoretical advantages and drawbacks in helping clinicians to optimise treatment strategies. However, none of these tools has really been prospectively validated in a study aimed at improving CD outcome. Therefore their use in the monitoring of patients with $\mathrm{CD}$ in routine practice remains empirical. A proposed algorithm of CD monitoring based on the limited available evidence and our experience is shown in figure 4 . The standard of care in CD is still to manage patients according to disease evolution based on clinical symptoms. However, on a case by case basis, monitoring can be used to optimise therapeutic strategy, aiming at decreasing cumulative tissue damage and its consequences. This could be particularly adapted in situations where there is concern about disease progression, because of disease location and extent, or history of the patients. In this case, the use of endoscopy, cross-sectional imaging and biomarkers should be proposed, aiming to answer specific questions leading to treatment adaptation. Biomarkers such as blood 


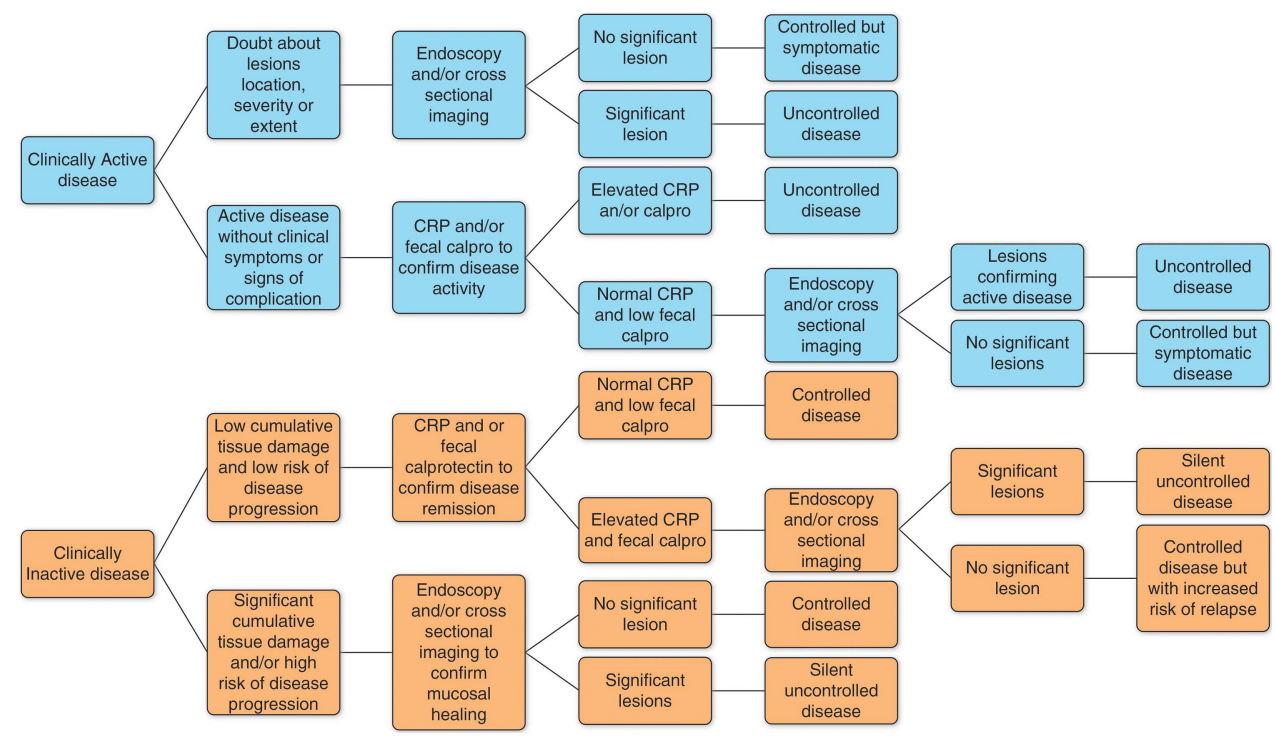

Figure 4 Proposed algorithm for Crohn's disease (CD) monitoring based on the limited available evidence (see also tables 4 and 5 ) and our experience. It illustrates how CD monitoring can affect disease management. In the presence of only two clinical situations (clinically active or inactive disease), CD monitoring can reveal five very different situations: controlled disease, symptomatic but controlled disease, controlled disease but with increased risk of relapse, silent uncontrolled disease, uncontrolled disease. These five different situations will lead to different treatment strategies and further monitoring. A clear definition and criteria for significant and non-significant endoscopic or cross-sectional imaging lesions as well as of controlled and uncontrolled disease are lacking. Minor persisting endoscopic or cross-sectional imaging lesions and slightly raised faecal calprotectin (calpro) may still be compatible with controlled disease. This will be left to the clinician's discretion, after also taking into account the patient's history (cumulative tissue damage, response to treatment). CRP, C-reactive protein.

$\mathrm{CRP}$ and faecal calprotectin concentration often represent informative first-line tests to try to predict disease activity, tissue healing or the risk of relapse. By choosing the thresholds of these markers appropriately to optimise sensitivity or specificity, up to half of endoscopic or cross-sectional imaging procedures could be avoided. New biomarkers still in development, as well as blood drug levels, including trough levels of biological agents, may also improve the efficacy of monitoring in the future. Prospective studies to confirm the effect of monitoringbased therapeutic changes on key outcomes of CD are urgently required.

\section{Summary Box 1}

- Monitoring of Crohn's disease with endoscopy, cross-sectional imaging and biomarkers aims to achieve tighter disease control to try to prevent tissue damage, with an optimal benefit/risk and benefit/cost ratio.

- Mucosal healing assessed by ileocolonoscopy is associated with improved disease outcome including fewer relapses, hospitalisations and operations.

- The degree of mucosal healing required to improve outcome is not completely established.

- Cross-sectional imaging provides information on the small bowel and the transmural and extramural features of the inflammatory process.

- MRI score of activity correlates well with endoscopic score of activity.

- Faecal calprotectin and blood C-reactive protein, can predict mucosal healing and relapse of the disease with an accuracy of $60-70 \%$.

\section{Summary Box 2}

- No prospective or even retrospective study has validated a treatment strategy based on disease monitoring aimed at improving disease outcome.

- Crohn's disease monitoring with endoscopy, cross-sectional imaging and biomarkers can only be empirical and should be decided on a case by case basis.

- Patients at high risk of disease progression or having a rapidly aggressive disease are the best candidates for empirical disease monitoring.

- Preference should be given to non-invasive and cheap first-line tests such as biomarkers.

- In asymptomatic patients, the decision whether to perform endoscopic or cross-sectional imaging monitoring could be based on disease history and results of the biomarkers.

- Treatment should be empirically adapted according to monitoring results to optimise benefit/cost and benefit/risk for the patient.

Contributors J-MB and EL wrote the first draft of the manuscript. M-AM reviewed and amended the part on biomarkers. CR and CVK reviewed and amended the part on endoscopy. PM reviewed and amended the part on cross-sectional imaging. All authors approved the final manuscript.

Provenance and peer review Commissioned; externally peer reviewed.

\section{REFERENCES}

1 Peyrin-Biroulet L, Loftus EV Jr, Colombel JF, et al. The natural history of adult Crohn's disease in population-based cohorts. Am J Gastroenterol 2010;105:289-97. 
2 Cosnes J, Nion-Larmurier I, Beaugerie L, et al. Impact of the increasing use of immunosuppressants in Crohn's disease on the need for intestinal surgery. Gut 2005:54:237-41.

3 Frøslie KF, Jahnsen J, Moum BA, et al. Mucosal healing in inflammatory bowe disease: results from a Norwegian population-based cohort. Gastroenterology 2007; 133:412-22

4 Schnitzler $F$, Fidder $H$, Ferrante $M$, et al. Mucosal healing predicts long-term outcome of maintenance therapy with infliximab in Crohn's disease. Inflamm Bowe Dis 2009;15:1295-301.

5 Baert F, Moortgat L, Van Assche G, et al. Mucosal healing predicts sustained clinical remission in patients with early-stage Crohn's disease. Gastroenterology 2010;138:463-8.

6 Rutgeerts P, Van Assche G, Sandborn W J, et al. Adalimumab induces and maintains mucosal healing in patients with Crohn's disease: data from the EXTEND trial. Gastroenterology 2012;142:1102-11.

7 Cellier C, Sahmoud T, Froguel E, et al. Correlations between clinical activity, endoscopic severity, and biological parameters in colonic or ileocolonic Crohn's disease. A prospective multicentre study of 121 cases. The Groupe d'Etudes Therapeutiques des Affections Inflammatoires Digestives. Gut 1994;35:231-5.

8 Panes J, Bouzas R, Chaparro M, et al. Systematic review: the use of ultrasonography, computed tomography and magnetic resonance imaging for the diagnosis, assessment of activity and abdominal complications of Crohn's disease. Aliment Pharmacol Ther 2011:34:125-45.

9 Lewis JD. The utility of biomarkers in the diagnosis and therapy of inflammatory bowel disease. Gastroenterology 2011;140:1817-26.

10 Langhorst J, Kühle CA, Ajaj W, et al. MR colonography without bowel purgation for the assessment of inflammatory bowel diseases: diagnostic accuracy and patient acceptance. Inflamm Bowel Dis 2007;13:1001-8.

11 Terheggen G, Lanyi B, Schanz S, et al. Safety, feasibility, and tolerability of ileocolonoscopy in inflammatory bowel disease. Endoscopy 2008;40:656-63.

12 Samuel S, Bruining DH, Loftus EV Jr, et al. Endoscopic skipping of the distal terminal ileum in Crohn's disease can lead to negative results from ileocolonoscopy. Clin Gastroenterol Hepatol 2012;10:1253-9.

13 Colombel J F, Sandborn W J, Reinisch W, et al. Infliximab, azathioprine, or combination therapy for Crohn's disease. N Engl J Med 2010;362:1383-95.

14 Allez M, Lemann M, Bonnet J, et al. Long term outcome of patients with active Crohn's disease exhibiting extensive and deep ulcerations at colonoscopy. Am J Gastroenterol 2002;97:947-53.

15 Prajapati J. Symptomatic luminal strictures underlies infliximab non response in inflammatory bowel disease. Gastroenterology 2002;122:A777

16 Mary J Y, Modigliani R. Development and validation of an endoscopic index of the severity for Crohn's disease: a prospective multicentre study. Groupe d'Etudes Therapeutiques des Affections Inflammatoires du Tube Digestif (GETAID). Gut 1989;30:983-9.

17 Daperno M, D'Haens G, Van Assche G, et al. Development and validation of a new, simplified endoscopic activity score for Crohn's disease: the SES-CD. Gastrointest Endosc 2004;60:505-12

18 Hanauer S B, Feagan B G, Lichtenstein G R, et al. Maintenance infliximab for Crohn's disease: the ACCENT I randomised trial. Lancet 2002;359:1541-9.

19 Baert F, Moortgat L, Van Assche G, et al. Mucosal healing predicts sustained clinical remission in patients with early-stage Crohn's disease. Gastroenterology 2010;138:463-8.

20 Landi B, Anh TN, Cortot A, et al. Endoscopic monitoring of Crohn's disease treatment: a prospective, randomized clinical trial. The Groupe d'Etudes Therapeutiques des Affections Inflammatoires Digestives. Gastroenterology 1992:102:1647-53.

21 Louis E, Mary J Y, Vernier-Massouille G, et al. Maintenance of remission among patients with Crohn's disease on antimetabolite therapy after infliximab therapy is stopped. Gastroenterology 2012;142:63-70.

22 Rutgeerts P, Geboes K, Vantrappen G, et al. Predictability of the postoperative course of Crohn's disease. Gastroenterology 1990;99:956-63.

23 Bourreille A, Ignjatovic A, Aabakken L, et al. Role of small-bowel endoscopy in the management of patients with inflammatory bowel disease: an international OMED-ECCO consensus. Endoscopy 2009:41:618-37.

24 Jensen MD, Nathan T, Rafaelsen SR, et al. Diagnostic accuracy of capsule endoscopy for small bowel Crohn's disease is superior to that of MR enterography or CT enterography. Clin Gastroenterol Hepatol 2011:9:124-9.

25 Van Assche G, Dignass A, Panes J, et al. The second European evidence-based consensus on the diagnosis and management of Crohn's disease: definitions and diagnosis. J Crohns Colitis 2010:4:7-27.

26 Horsthuis K, Bipat S, Stokkers PC, et al. Magnetic resonance imaging for evaluation of disease activity in Crohn's disease: a systematic review. Eur Radiol 2009;19:1450-60.

27 Pariente B, Peyrin-Biroulet L, Cohen L, et al. Gastroenterology review and perspective: the role of cross-sectional imaging in evaluating bowel damage in Crohn disease. Am J Roentegenol 2011;197:42-9.

28 Maconi G, Radice E, Greco S, et al. Bowel ultrasound in Crohn's disease. Best Pract Res Clin Gastroenterol 2006:20:93-112.
29 Bru C, Sans M, Defelitto MM, et al. Hydrocolonic sonography for evaluating inflammatory bowel disease. Am J Roentgenol 2001;177:99-105.

30 Rimola J, Rodríguez S, García-Bosch 0, et al. Magnetic resonance for assessment of disease activity and severity in ileocolonic Crohn's disease. Gut 2009;58:1113-20.

31 Rimola J, Ordás I, Rodríguez $\mathrm{S}$, et al. Magnetic resonance imaging for evaluation of Crohn's disease: validation of parameters of severity and quantitative index of activity. Bowel Dis 2011;17:1759-67.

32 Zappa M, Stefanescu C, Cazals-Hatem D, et al. Which magnetic resonance imaging findings accurately evaluate inflammation in small bowel Crohn's disease? A retrospective comparison with surgical pathologic analysis. Inflamm Bowel Dis 2011;17:984-93.

33 Punwani S, Rodríguez-Justo M, Bainbridge A, et al. Mural inflammation in Crohn disease: location-matched histologic validation of MR imaging features. Radiology 2009:252:712-20.

34 Gourtsoyiannis NC, Papnikolaou N, Karantanas A. Magnetic resonance imaging evaluation of small intestinal Crohn's disease. Best Pract Res Clin Gastroenterol 2006;20:137-56.

35 Siddiki HA, Fidler JL, Fletcher JG, et al. Prospective comparison of state-of-the-art MR enterography and CT enterography in small-bowel Crohn's disease. Am J Roentgenol 2009;193:113-21.

36 Lee SS, Kim AY, Yang SK, et al. Crohn disease of the small bowel: comparison of CT enterography, MR enterography, and small-bowel follow-through as diagnostic techniques. Radiology 2009;251:751-61.

37 Paredes JM, Ripolles T, Cortes $X$, et al. Abdominal sonographic changes after antibody to tumor necrosis factor alpha therapy in Crohn's disease. Dig Dis Sci 2010;55:404-10

38 Migaleddu V, Scanu AM, Quaia E, et al. Contrast-enhanced ultrasonographic evaluation of inflammatory activity in Crohn's disease. Gastroenterology 2009;137:43-52

39 Sempere J, Martínez-Sanjuan V, Medina Chulia E, et al. MRI evaluation of inflammatory activity in Crohn's disease. Am J Roentegenol 2005;184:1829-35.

40 Ordás I. Accuracy of MRI to assess therapeutic responses and mucosal healing in Crohn's diasease. Gastroenterology 2011;140:S73

41 Pariente B, Peyrin-Biroulet L, Cohen L, et al. Gastroenterology review and perspective: the role of cross-sectional imaging in evaluating bowel damage in Crohn disease. Am J Roentegenol 2011;197:42-9.

42 Giusti S, Faggioni L, Neri E, et al. Dynamic MRI of the small bowel: usefulness of quantitative contrast-enhancement parameters and time-signal intensity curves for differentiating between active and inactive Crohn's disease. Abdom Imaging 2010;35:646-53.

43 Lee SS, Kim AY, Yang SK, et al. Crohn disease of the small bowel: comparison of CT enterography, MR enterography, and small-bowel follow-through as diagnostic techniques. Radiology 2009:251:751-61.

44 Horsthuis K, Bipat S, Bennink RJ, et al. Inflammatory bowel disease diagnosed with US, MR, scintigraphy, and CT: meta-analysis of prospective studies. Radiology 2008;247:64-79.

45 Maconi G, Sampietro GM, Parente F, et al. Contrast radiology, computed tomography and ultrasonography in detecting internal fistulas and intraabdominal abscesses in Crohn's disease: a prospective comparative study. Am J Gastroenterol 2003;98:1545-55.

46 Koilakou S, Saliler J, Peloschek P, et al. Endoscopy and MR enteroclysis: equivalent tools in predicting clinical recurrence in patients with Crohn's disease after ileocolic resection. Inflamm Bowel Dis 2010;16:198-203.

47 Sailer J, Peloschek P, Reinisch W, et al. Anastomotic recurrence of Crohn's disease after ileocolic resection: comparison of MR enteroclysis with endoscopy. Eur Radiol 2008; 18:2512-21.

48 Pariente B, Cosnes J, Danese S, et al. Development of the Crohn's disease digestive damage score, the Lémann score. Inflamm Bowel Dis 2011;17:1415-22.

49 Pepys MB, Baltz ML. Acute phase proteins with special reference to C-reactive protein and related proteins (pentaxins) and serum amyloid A protein. Adv Immunol 1983:34:141-212.

50 Peyrin-Biroulet L, Gonzalez F, Dubuquoy L, et al. Mesenteric fat as a source of C reactive protein and as a target for bacterial translocation in Crohn's disease. Gut 2012;61:78-85

51 Ramadori G, Christ B. Cytokines and the hepatic acute-phase response. Semin Live Dis 1999;19:141-55.

52 Nacken W, Roth J, Sorg C, et al. Myeloid representatives of the S100 protein family as prominent players in innate immunity. Microsc Res Tech 2003;60:569-80.

53 Poullis A, Foster R, Mendall MA, et al. Emerging role of calprotectin in gastroenterology. J Gastroenterol Hepatol 2003;18:756-62.

54 Louis $E$, Vermeire $S$, Rutgeerts $P$, et al. A positive response to infliximab in Crohn disease: association with a higher systemic inflammation before treatment but not with -308 TNF gene polymorphism. Scand J Gastroenterol 2002:37:818-24.

55 Denis M A, Reenaers C, Fontaine F, et al. Assessment of endoscopic activity index and biological inflammatory markers in clinically active Crohn's disease with normal C-reactive protein serum level. Inflamm Bowel Dis 2007:13:1100-5. 
56 Sipponen T, Karkkainen P, Savilahti $\mathrm{E}$, et al. Correlation of faecal calprotectin and lactoferrin with an endoscopic score for Crohn's disease and histological findings. Aliment Pharmacol Ther 2008;28:1221-9.

57 Bondjemah V, Mary JY, Jones J, et al. Fecal calprotectin and CRP as biomarkers of endoscopic activity in Crohn's disease: a meta-study. J Crohn Colitis 2012;6(1): P133.

58 Sipponen $\mathrm{T}$, Bjorkesten C G, Farkkila $\mathrm{M}$, et al. Faecal calprotectin and lactoferrin are reliable surrogate markers of endoscopic response during Crohn's disease treatment. Scand J Gastroenterol 2010;45:325-31.

59 Jürgens M, Mahachie John JM, Cleynen I, et al. Levels of C-reactive protein are associated with response to infliximab therapy in patients with Crohn's disease. Clin Gastroenterol Hepatol 2011;9:421-7.

60 Schoepfer A M, Beglinger C, Straumann A, et al. Fecal calprotectin correlates more closely with the Simple Endoscopic Score for Crohn's disease (SES-CD) than CRP blood leukocytes, and the CDAI. Am J Gastroenterol 2010;105:162-9.

61 Jones J, Loftus E V Jr, Panaccione R, et al. Relationships between disease activity and serum and fecal biomarkers in patients with Crohn's disease. Clin Gastroenterol Hepatol 2008;6:1218-24.

62 Wagner $\mathrm{M}$, Peterson $\mathrm{CG}$, Ridefelt $\mathrm{P}$, et al. Fecal markers of inflammation used as surrogate markers for treatment outcome in relapsing inflammatory bowel disease. World J Gastroenterol 2008;14:5584-9.

63 D'Haens G, Ferrante M, Vermeire S, et al. Fecal calprotectin is a surrogate marker for endoscopic lesions in inflammatory bowel disease. Inflamm Bowel Dis 2012;18:2218-24.

64 Colombel JF, Solem CA, Sandborn WJ, et al. Quantitative measurement and visual assessment of ileal Crohn's disease activity by computed tomography enterography: correlation with endoscopic severity and $C$ reactive protein. Gut 2006;55:1561-7.

65 Jones J, Loftus EV Jr, Panaccione R, et al. Relationships between disease activity and serum and fecal biomarkers in patients with Crohn's disease. Clin Gastroenterol Hepatol 2008;6:1218-24
66 Lemann M, Colombel J-F, Grimaud J-C, et al. Fecal calprotectin and high sensitivity c-reactive protein levels to predict mucosal healing in patients with Crohn's disease. A subanalysis of the STORI study. Gut 2010;59(uppl III):A80.

67 Garcia-Sanchez V, Iglesias-Flores E, Gonzalez R, et al. Does fecal calprotectin predict relapse in patients with Crohn's disease and ulcerative colitis? J Crohns Colitis 2010;4:144-52

68 Kallel L, Ayadi I, Matri S, et al. Fecal calprotectin is a predictive marker of relapse in Crohn's disease involving the colon: a prospective study. Eur J Gastroenterol Hepatol 2010;22:340-5.

69 Tibble J A, Sigthorsson G, Bridger S, et al. Surrogate markers of intestinal inflammation are predictive of relapse in patients with inflammatory bowel disease. Gastroenterology 2000;119:15-22.

70 Consigny Y, Modigliani R, Colombel J F, et al. A simple biological score for predicting low risk of short-term relapse in Crohn's disease. Inflamm Bowel Dis 2006;12:551-7.

71 Koelewijn C L, Schwartz M P, Samsom M, et al. C-reactive protein levels during a relapse of Crohn's disease are associated with the clinical course of the disease. World J Gastroenterol 2008;14:85-9.

72 Bitton A, Dobkin PL, Edwardes MD, et al. Predicting relapse in Crohn's disease: a biopsychosocial model. Gut 2008;57:1386-92.

73 De Suray N, Salleron J, Vernier-Massouille G, et al.Close monitoring of CRP and fecal calprotectin levels to predict relapse in Crohn's disease patients. A sub-analysis of the STORI study. J Crohn Colitis 2012;6(1):P274.

74 Treton $X$, Bouhnik Y, Mary JY, et al. Azathioprine withdrawal in patients with Crohn's disease maintained on prolonged remission: a high risk of relapse. Clin Gastroenterol Hepatol 2009;7:80-5.

75 Regueiro M, Kip KE, Schraut W, et al. Crohn's disease activity index does not correlate with endoscopic recurrence one year after ileocolonic resection. Inflamm Bowel Dis 2011;17:118-26.

76 Lamb CA, Mohiuddin MK, Gicquel J, et al. Faecal calprotectin or lactoferrin can identify postoperative recurrence in Crohn's disease. Br J Surg 2009;96:663-74. 